\title{
SCIENTIFIC

\section{OPEN Reduced holey graphene oxide film and carbon nanotubes sandwich structure as a binder-free electrode material for supercapcitor}

\author{
Khan Abdul Sammed ${ }^{1}$, Lujun Pan ${ }^{1 *}$, Muhammad Asif ${ }^{2}$, Muhammad Usman ${ }^{1,4}$, Tianze Cong ${ }^{1}$, \\ Farid Amjad ${ }^{1}$ \& Muhammad Asif Imran ${ }^{3}$
}

A novel carbon nanotubes (CNTs) and reduced holey graphene oxide film (RHGOF) sandwich structure has been fabricated to enhance its electrochemical properties. CNTs are grown by a catalyst assisted chemical vapor deposition technique, interpenetrated between the RHGOF layers. A RHGOF/CNTs hybrid film is used as a binder-free supercapacitor electrode. The grown CNTs in the graphene layers structure act as spacers and bridges to increase the counductivity of RHGOF, while the grown CNTs on the surfaces of the graphene contribute to increase the specific surface area of RHGOF. The results demonstrate that the synthesized porous, flexible and binder free hybrid electrode has advantages of higher ion diffusion rate, longer diffusion length and larger ion accessible surface area as compared to the pristine graphene which results in an extra ordinary galvanostatic charge-discharge specific capacitance of $557 \mathrm{~F} / \mathrm{g}$ at a current density of $0.5 \mathrm{~A} / \mathrm{g}$, with excellent rate capabilities and superior cyclic stabilities.

In recent era, environment adaptability, portability and compatibility of electronics, hybrid electric vehicles are becoming challenging issues. Energy storage devices having high performance are the part and parcel of these aforementioned devices ${ }^{1}$. Because of its promising adventages, supercapacitor is one of the fascinating energy storage device, such as fast dynamic response, high power density, longer life span and good capability rate ${ }^{2,3}$. Therefore, explicit studies of the characteristics of supercapcitor electrode materials are very essential components that ultimately affect its electrochemical properties ${ }^{1,4-7}$. Most recently, researchers are trying their best to prepare flexible type of electrode materials possessing good cyclic stability, high performance output in terms of charge-discharge rates and specific capacitance.

Graphene (GR), being a 2D nano-structured material, has already been acknowledged as an attractive material for supercapacitor electrode. As a single thick sheet of carbon atoms, due to the numerous profound physic-chemical properties, it has been presaged for transfiguring a wide range of technological area ${ }^{8-11}$. Moreover, it has high unique mechanical flexibility intrinsic electrical conductivity, theoretical gravimetric capacitance of about $550 \mathrm{~F} / \mathrm{g}$ and remarkably large theoretical surface area of $2630 \mathrm{~m}^{2} / \mathrm{g}^{11-13}$. On the contrary, due to the extended $\pi$-conjugation in the basal plane, pristine GR has van der Waals forces and $\pi-\pi$ interaction. Thus, GR sheets are stacked with each other under these effects. Their irreversible agglomeration not only hinders the ion diffusion rate, but also decreases accessible surface area and consequent reduction in the gravimetric capacitance ${ }^{14-17}$. To improve its capacitive performance, numerous methodologies have already been proposed. For instance, to prevent the restacking, conducting carbon materials as an active channel (such as carbon black, carbon nanotubes (CNTs)) and conducting polymers (such as polyaniline), have been used. Additionally, nanostructured pseudo capacitive materials (such as $\mathrm{MnO}_{2}$, and $\mathrm{Mn}_{3} \mathrm{O}_{4}$ etc.) were also used to enhance capacitive properties ${ }^{18-23}$.

Recently, CNT/GR hybrid materials have been realized as the most capable candidate for electrode materials because of their improved capacitive properties and high flexibility. Many studies on CNT/GR hybrids are reported in literature. For instance, Yan et al. synthesized CNT/GR hybrid materials on nickel foam by using a

${ }^{1}$ School of Physics, Dalian University of Technology, Dalian, 116024, PR of China. ${ }^{2}$ Department of Materials Science and Engineering College of Engineering, Peking University, Beijing, 100871, China. ${ }^{3}$ School of Chemical Engineering, Dalian University of Technology, Dalian, 116024, PR of China. ${ }^{4}$ Department of Physics, Khwaja Fareed University of Engg. and Information Technology, Rahimyar, Khan-64200, Pakistan. *email: Ipan@dlut.edu.cn 
two-step chemical vapor deposition (CVD) method and reported 104 F/g specific capacitance in their composite $^{24}$. Moreover Fan et al. produced CNT/GR hybrid composite by the arrangement of microwave methods and CVD technique that shown high specific capacitance of $385 \mathrm{~F} / \mathrm{g}$ at $10 \mathrm{mV}$ scan rate ${ }^{25}$. However mostly GR/CNT hybrid electrode composites were synthesized by using binders because they were in the form of powder. Mean while, Xiong et al. adopted the combination of floating catalyst CVD and electrophoretic deposition that led to the preparation of GR/CNT/nickel foam hybrid materials, having promising $236.18 \mathrm{~F} / \mathrm{g}$ specific capacitance. Although this composite can be used directly as supercapacitor electrode ${ }^{26}$, the GR/CNT cannot be used independently without the support of Ni foam. Therefore, currently free-standing flexible CNT/GR composite with adequate capacitive properties is the need of hour to extend its applications. For free standing, Fan et al. used polystyrene colloidal particles as template and synthesized 3D porous nitrogen doped GR/CNT flexible paper to enhance capacitive properties ${ }^{27}$. They reported specific capacitance of $294 \mathrm{~F} / \mathrm{g}$ at $1 \mathrm{~A} / \mathrm{g}$ current density in $6 \mathrm{M}$ $\mathrm{KOH}$ aqueous electrolyte.

Even though the already published GR/CNT film electrodes have shown good capacitive properties, still these characteristics are limited because of the $\pi-\pi$ stacking of GR. Hence synthesizing holey graphene (HGR) is a rational idea to remove $\pi-\pi$ stacking and produce good capability rates and shorten the ion diffusion path ${ }^{28-31}$. For instance, Yu et al. prepared HGR oxide and its derivative macrostructures which exhibited significantly higher accessible surface area as well as ion diffusion rate as compared to non-holey composites ${ }^{28}$. These binder free porous composites were used directly as supercapacitor components, which displayed the specific capacitance of $283 \mathrm{~F} / \mathrm{g}$. Cheng et al. prepared flexible and porous HGR paper by using vacuum filtration technique. This paper was used directly as supercapacitor electrodes, exhibiting a specific capacitance of $201 \mathrm{~F} / \mathrm{g}$ at $1 \mathrm{~A} / \mathrm{g}$ with excellent cyclic stabilities and good rate capabilities ${ }^{32}$. Deng et al. prepared a CNT/HGR flexible free standing film by using vacuum filtration of the mixed functional CNTs and HGR solutions, the prepared CNT/HGR hybrid electrode material exhibited an ultra-high specific capacitance of $268 \mathrm{~F} / \mathrm{g}$ at $0.25 \mathrm{~A} / \mathrm{g}$ with superior cyclic stabilities and excellent rate capabilities. Although their composite showed a good output but CNTs are agglomerated during the solution based approach. Hence, whole area of HGR as substrate cannot be utilized ${ }^{33}$.

To address the aforementioned concerns, a facile method is devised to synthesize CNTs and reduced holey graphene oxide film (RHGOF) hybrid multi-layered sandwich structure as a binder-free electrode by the combination of vacuum filtration technique and CVD. This 3D structure is not only supportive to accelerate electron transfer during charge-discharge process but also beneficial in utilizing specific surface area to a greater extent.

\section{Characterization}

Electrochemical characterizations. Electrochemical performances of the samples were analyzed by Galvanostatic charge-discharge (CD), cyclic voltammetry (CV), and electrochemical impedance spectroscopy (EIS). Electrochemical work station (CHI660E instruments Inc. China) was used to carry out aforementioned characterization by using three electrode system, which includes $\mathrm{Hg} / \mathrm{HgO}$ as a reference electrode, a piece of $\mathrm{Pt}$ as counter electrode, and as prepared binder free flexible electrode (RHGOF/CNTs) as working electrode in a $6 \mathrm{M} \mathrm{KOH}$ electrolyte solution. This system was used to measure $\mathrm{CV}$ and $\mathrm{CD}$ at scan rates of 5 to $100 \mathrm{mV} / \mathrm{s}$ under a potential window from -0.9 to $-0.1 \mathrm{~V}$ and at different current densities from 0.5 to $6 \mathrm{~A} / \mathrm{g}$, respectively. The specific capacitance of electrodes was calculated from CD curves through the following formula.

$$
\mathrm{C}=\frac{\mathrm{It}}{\Delta \mathrm{V} \cdot \mathrm{m}}
$$

where constant discharge current $(\mathrm{A})$ is represented by I, mass of active material ( $\mathrm{g}$ ) by $\mathrm{m}$ and the $\mathrm{t}$ shows the time of discharge stage $(\mathrm{s})$ and $\Delta \mathrm{V}$ is the potential difference of charging $(\mathrm{V})$. Time relaxation constant was measured by the following equation.

$$
t_{o}=\frac{1}{f_{o}}
$$

whereas, $f_{0}$ represents characteristic frequency and $\mathrm{t}_{0}$ is time relaxation constant. At a phase angle of $-45^{\circ}, \mathrm{t}_{0}$ stands for the minimum time needed to discharge all the energy from the device with an efficiency of more than $50 \%$.

\section{Results and Discussion}

The formation scheme of binder-free sandwich structure containing RHGOF and CNTs is illustrated in Fig. 1. The GO was etched by hydrothermal reaction with $\mathrm{H}_{2} \mathrm{O}_{2}$ at low temperature and was reduced with hydrazine, respectively. Final suspension was filtered out in order to get flexible RHGO film. Later on, RHGOF/CNTs sandwich structure was obtained after applying CVD method.

Actually, GO nano sheets possess numerous active sites. These sites were further etched to generate porous carbon vacancies on the surface. The porous structure of the sample was confirmed by nitrogen desorption/ adsorption analysis. Nitrogen adsorption/desorption tests showed the RHGOF exhibited a much higher Brunauer-Emmett-Teller specific surface area of $\sim 560.8 \mathrm{~m}^{2} / \mathrm{g}$ than RGOF $\left(\sim 254.6 \mathrm{~m}^{2} / \mathrm{g}\right)$ and a BarrettJoyner-Halenda average pore size distribution is $3.25 \mathrm{~nm}$. (Surface area data are shown in the Supporting Information). It is observed that the surface area of graphene within the RHGOF is highly accessible due to the nanopores in the basal plan inspite of the compact stacking structure. Particularly, the nanopores in RHGOF not only promoted ion diffusion and access to the graphene surface than in RGOF nanosheets ${ }^{28}$, it also provides a short pathways for electrolyte to penetrate inside the layers that directly enhance the accessible surface area.

Moreover due to the sandwich structure of RHGOF/CNTs composite, CNTs acting as spacer and provides conducting bridges in RHGOF/CNTs. Hence larger accessible surface areas are offered. More electron and ion 

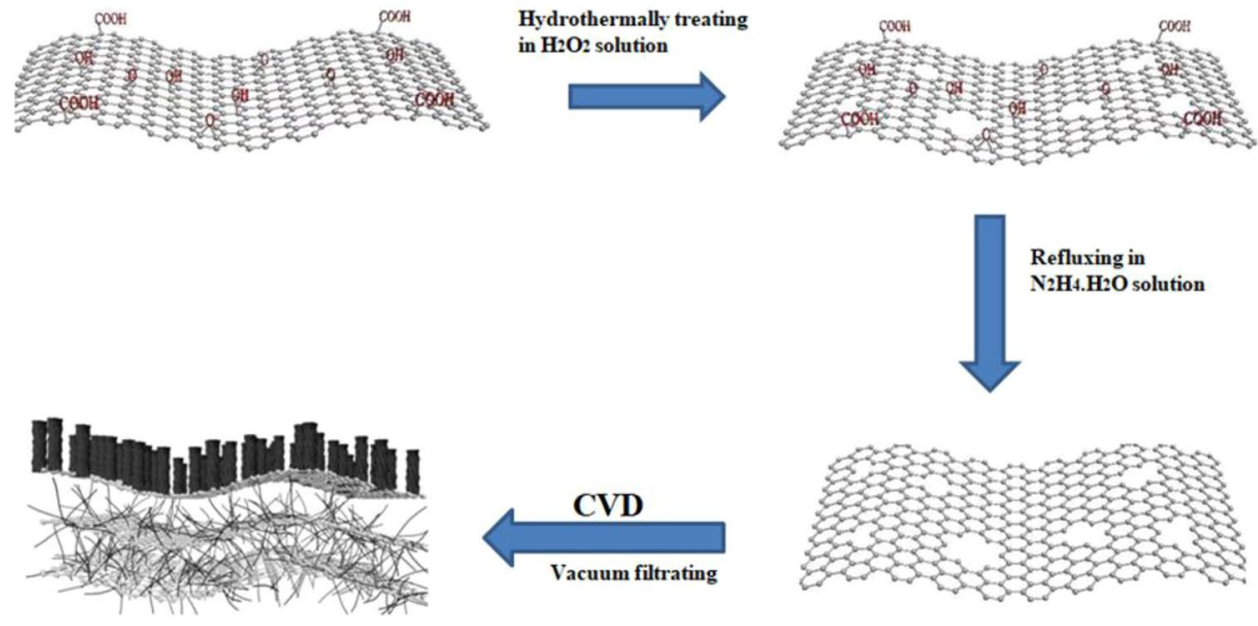

Figure 1. Formation scheme of RHGOF/CNTs sandwich structure.
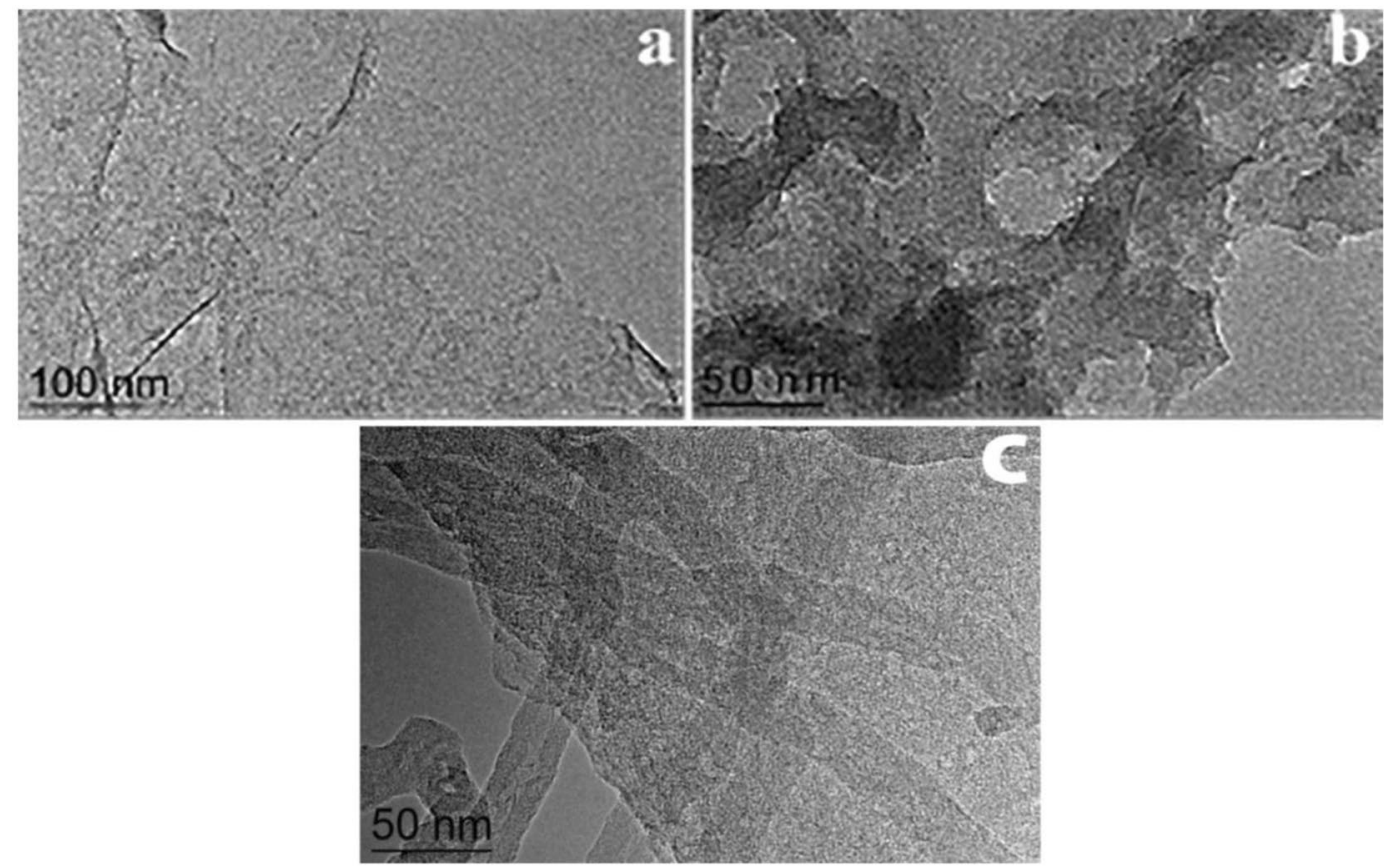

Figure 2. (a) TEM image RGOF (b) TEM image of RHGOF, (c) TEM image of RHGOF/CNTs hybrid composite.

transfer pathways are generated in favor of the electrolyte penetration. The obtained flexural strength (๘) and modulus of elasticity(E) of RHGOF/CNTs are 4.4 TPa and 5.21 TPa respectively. These values are significantly higher than published ${ }^{34}$.

Figure 2a, TEM image of RGOF that reveals very stable nature of transparent sheets.

While, RHGOF analysis is evidenced as non-transparent sheets morphology mainly due to the efficient etching of GO nanosheets by $\mathrm{H}_{2} \mathrm{O}_{2}$. Moreover, it also exhibited that across the basel plane of 2D HGO, there is enriched in-plane pores with ranging few nanometers size (Fig. 2b). In Fig. 2c TEM of RHGOF/CNTs clearly shows that surface of HGR sheet was combined with CNTs significantly, having an average diameter of $24 \mathrm{~nm}$. Additionally, Fig. $2 \mathrm{c}$ reveals that in flexible films CNTs serve as a physical spacer. Hence, a precise porous sandwich structure is formed, which provided enough pathways to electrolyte between the HGR sheets. This sandwich structure RHGOF/CNTs is considered to be a more promising super-electrochemical capacitor (SEC) electrode material as compared to RGOF and RHGOF. Because interlayer space between the nanosheets of RGOF and RHGOF was too small to penetrate the electrolyte as compared to RHGOF/CNTs ${ }^{35-37}$.

FESEM images of prepared samples (RGOF, RHGOF and RHGOF/CNTs) are shown in Fig. 3. Figure 3a shows RGOF no flakes on the surface, rather reveal grooves. These fluctuations might be caused by overlaping of GO nanosheets causing aggregations during the filtration process. 

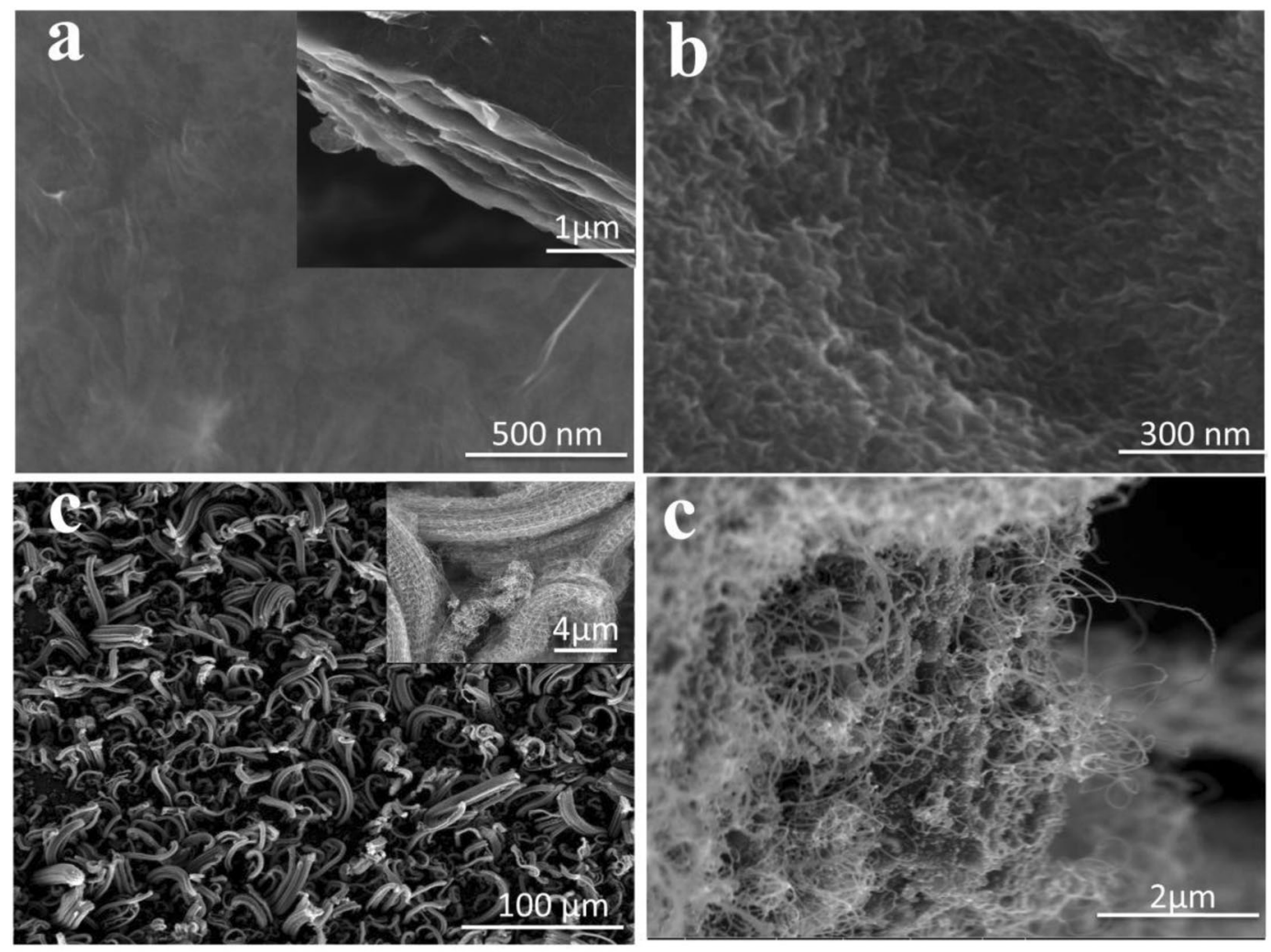

Figure 3. (a) FESEM images of RGOF, cross-sectional image of RGOF inset of (a,b) RHGOF, (c) RHGOF/ CNTs hybrid composite, magnified image of RHGOF/CNTsis inset of (c,d) Cross-sectional image of RHGOF/ CNTs.

The cross-section of RGOF paper elucidating layered structure that was also reported in the published research $^{27}$ (Fig. 3a inset). On the other hand, FESEM of RHGOF shows porous and flake-like structures. During filtration process flake-like graphene nanosheets are assembled that formed a flexible and compact layered structures with larger specific surface area as compared to RGOF (Fig. 3b). In this work, CNTs were grown through the CVD method. Figure $3 \mathrm{c}$ depicts bundles of CNTs on the upper surface of the RHGOF. Moreover, cross-section of hybrid RHGOF/CNTs reveals that strings of CNTs were grown inside the layers (Fig. 3d). This is due to exposure of large surface area to the $\mathrm{C}_{2} \mathrm{H}_{2}$ and Ar to do the promising reaction at upper surface of the film. Preceding more it is found that, bundles were uniformly distributed along the surface. Inset of Fig. $3 c$ shows high resolution image of CNTs bundles at the upper surface of the RHGOF film.

The chemical states and binding energies were identified and analyzed by XPS, as shown in Fig. 4a. It is confirmed from the survey image that the $\mathrm{C}$ and $\mathrm{O}$ are the main elements in the prepared RHGOF sample along with small percentage of $\mathrm{N}$ atoms. Figure $4 \mathrm{c}$ reveals that the $\mathrm{C} 1 \mathrm{~s}$ spectrum of RHGOF is constituted into three sub-peaks. These peaks centered at $288.05,285.05$ and $284.2 \mathrm{eV}$ corresponding to $\mathrm{C}=\mathrm{O}, \mathrm{C}-\mathrm{O}$ and $\mathrm{C}-\mathrm{C}$, bond resonances, respectively. The peak of $\mathrm{C}-\mathrm{C}$ bonds in $\mathrm{C} 1 \mathrm{~s}$ core-level spectrum relates to the $\mathrm{sp}^{2}$ hybridized carbon, represents carbon material, where as, $\mathrm{C}-\mathrm{O}$ and $\mathrm{C}=\mathrm{O}$ represent oxygen functionalities. $\mathrm{O} 1 \mathrm{~s}$ spectrum is divided into three different peaks at $533.0 \mathrm{eV}$ (COOH groups), $532.1 \mathrm{eV}$ (phenolic $\mathrm{C}-\mathrm{OH}$ or $\mathrm{C}-\mathrm{O}-\mathrm{C}$ bonds) and $530.8 \mathrm{eV}$ (quinonoid $\mathrm{C}=\mathrm{O}$ bonds) as shown in Fig. $4 \mathrm{~b}^{38}$. These oxygen-functional groups are responsible for reversible pseudo-capacitive reactions.

In order to exposing the defects in the as prepared samples (RGOF, RHGOF and RHGOF/CNTs), Raman spectroscopy study is carried out, as shown in the Fig. $4 \mathrm{~d}$. The $\mathrm{D}$ band is ascribed to the $\mathrm{sp}^{3}$ hybridized carbon atoms and it reveals the defects is generated in the graphene basal plane ${ }^{39}$ and in graphitic materials first order scattering mode of $\mathrm{sp}^{2}$ hybridized carbon atoms within the lattice plane of the graphene materials correspond to G band ${ }^{40}$.

All three samples show different $\mathrm{D}$ and $\mathrm{G}$ band, $\left(\mathrm{I}_{\mathrm{D}} / \mathrm{I}_{\mathrm{G}}\right)$ intensities ratios as shown in the Fig. $4 \mathrm{~d}$. From Fig. $4 \mathrm{~d}$, it is shown that $I_{D} / I_{G}$ ratio of RHGOF is increased as compared to RGOF, which is resulted from the formations of abundant pores and defects in the basal plane of graphene. Similarly remarkable increase of $\mathrm{I}_{\mathrm{D}} / \mathrm{I}_{\mathrm{G}}$ ratio for RHGOF/CNTs composite as compared to aforementioned composites elucidates that more defects is generated due to the CNT growth.

As shown in Fig. 5 the reversible redox conversions take place in quinonoid $\mathrm{C}=\mathrm{O}$ bonds, phenolic $\mathrm{C}-\mathrm{OH}$ bonds and $\mathrm{COOH}$ groups, which provided the pseudo-capacitance effect in the porous oxygen-doped carbon electrodes. 

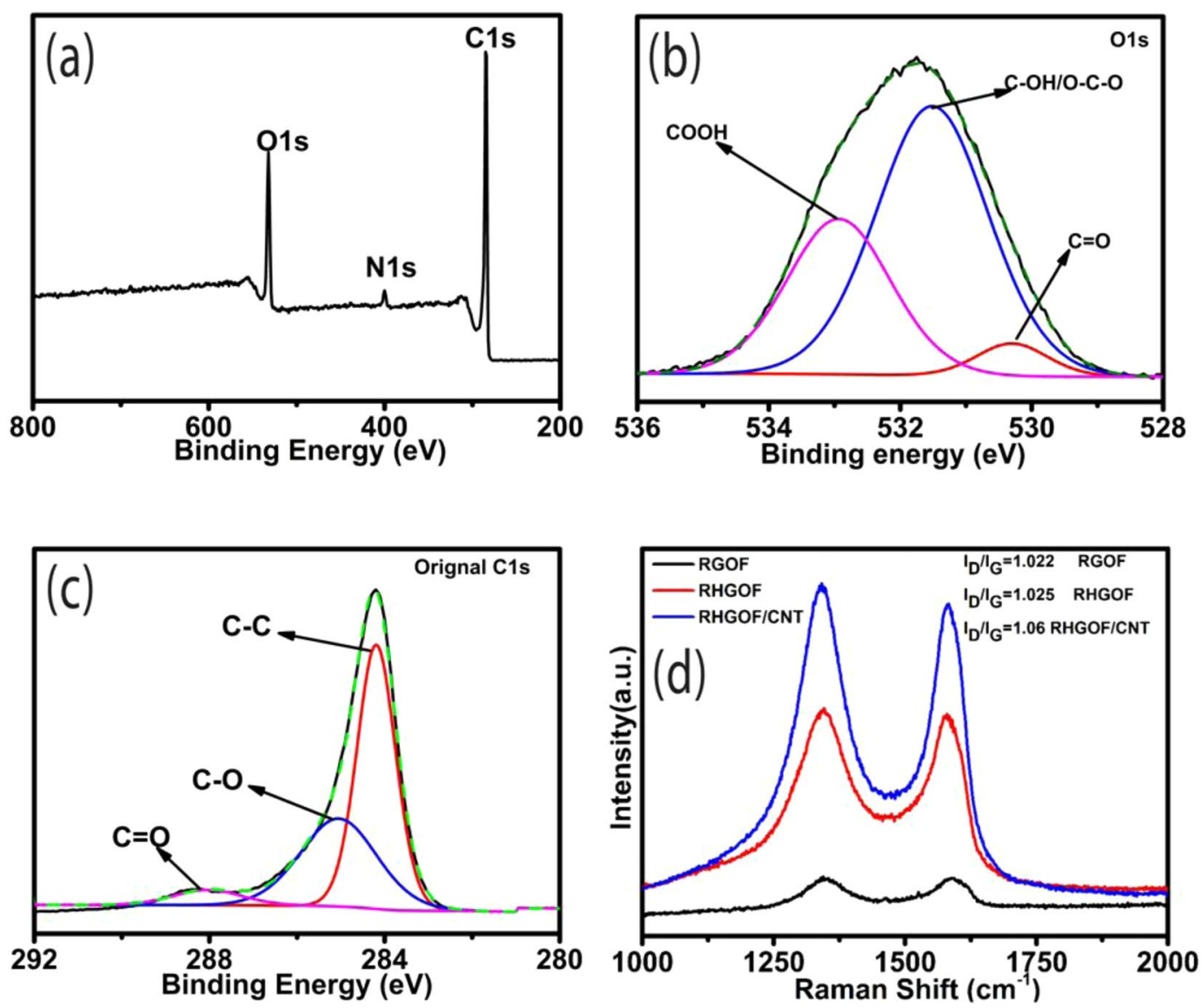

Figure 4. (a) XPS survey, (b) O1s spectra, and (c) C1s spectra for RHGOF. (d) Raman of RGOF, RHGOF and RHGOF/CNTs.
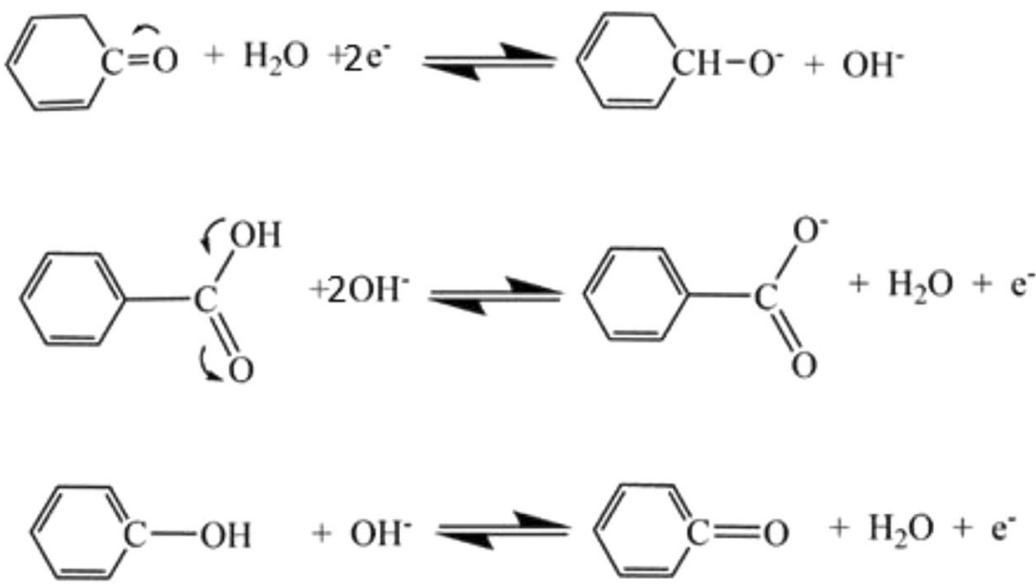

Figure 5. The various oxygen-functional groupsanticipated by reversible pseudo-capacitive reactions.

Capacitive properties of RGOF, RHGOF and RHGOF/CNTs hybrid electrodes were investigated in $6 \mathrm{M} \mathrm{KOH}$ aqueous electrolyte by CV, CD and EIS. CV curves of samples for RGOF, RHGOF, and RHGOF/CNTs are shown in Fig. $6 \mathrm{a}$, measured between $-0.1 \mathrm{~V}$ to $-0.9 \mathrm{~V}$ potential windows at the scan rate of $10 \mathrm{mV} / \mathrm{s}$. Figure $6 \mathrm{~b}$ depicts, the $\mathrm{CV}$ curves of the RHGOF/CNTs hybrids at different scan rates.

The designed hybrid structure, during the scan rates ranging from $5 \mathrm{mV}$ to $100 \mathrm{mV}$ displays outstanding electrochemical behavior, which implies that 3D RHGOF/CNTs flexible electrode shows a good rate capability. Likewise, comparison of areas of CV curves between RHGOF electrode and RGOF electrode shows RHGOF has superior area than that of RGOF. It is also shown from the Fig. 6a that the CV curve area of the RHGOF/CNTs electrode is greater than that of both aforementioned electrodes. Consequently, due to contribution of the large 

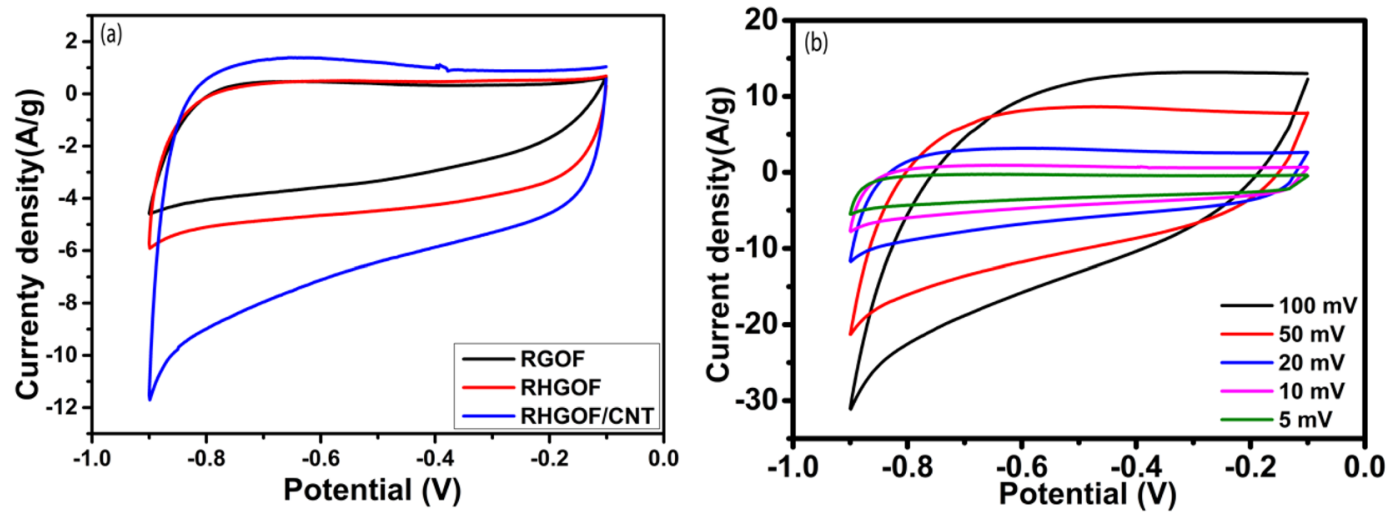

Figure 6. (a) CV curves of RGOF, RHGOF and RHGOF/CNTs at scan rate of $10 \mathrm{mV}$, (b) the CV curves of RHGOF/CNTs at different scan rates.
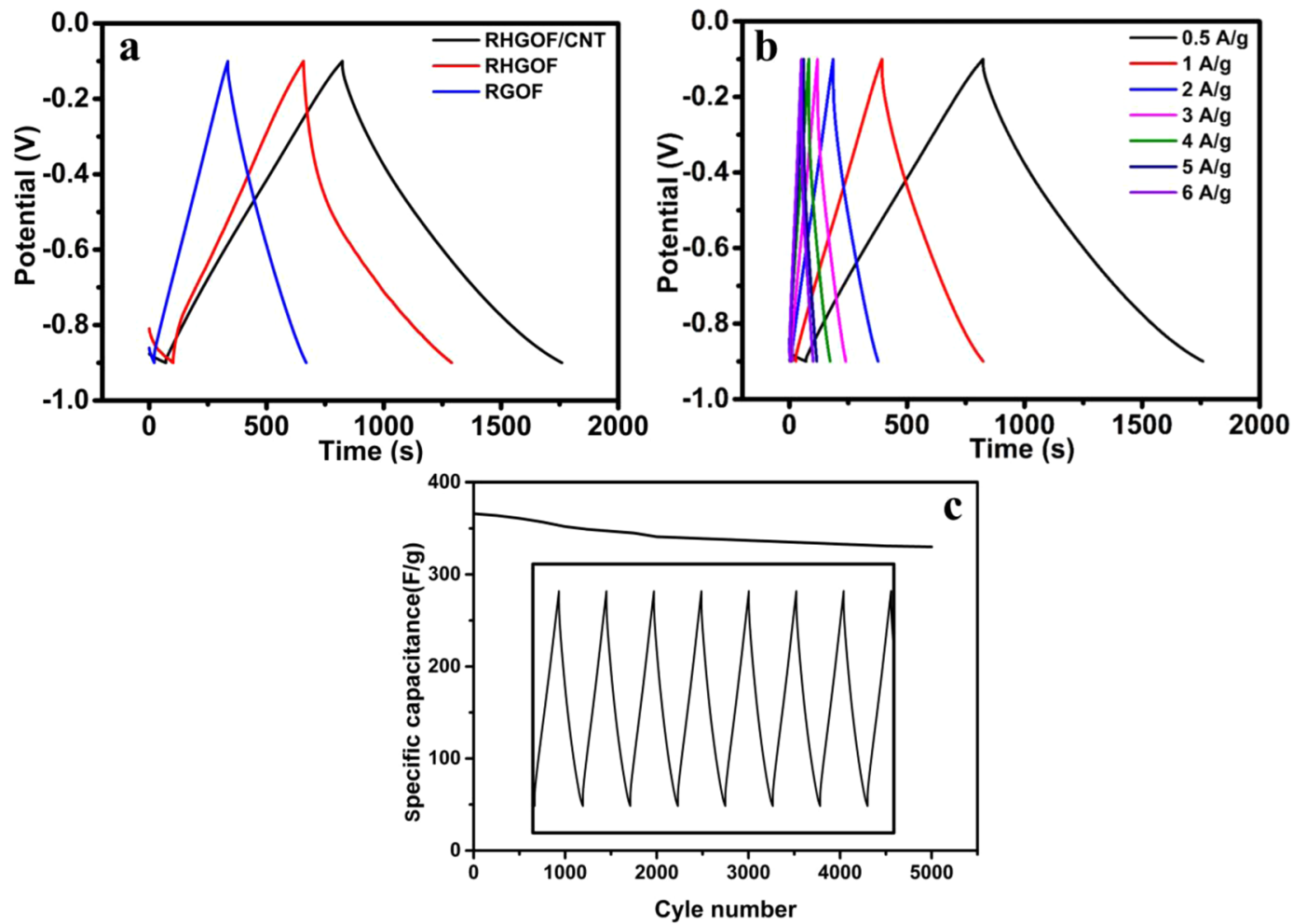

Figure 7. (a) CD curves of RGOF,RHGOF and RHGOF/CNTs at $0.5 \mathrm{~A} / \mathrm{g}$ current density, (b) at different current densities CD curves of RHGOF/CNTs (c) Cyclic stablity of RHGOF/CNTs.

integrated surface area, the specific capacitance is increased. In fact, due to the etching of graphene nano-sheets with a suitable amount of $\mathrm{H}_{2} \mathrm{O}_{2}$ during synthesis of RHGOF through hydrothermal process, the accessible specific surface area is increased. Also, it is shown that RHGOF/CNTs has largest accessible surface area as compared to the RGOF and RHGOF. It is evidenced that the CNTs grown within a sandwich structure act as a spacer, as confirmed by FESEM and TEM results. At the same scan rate, the improvement of cyclic voltammetry performance is attributed to the porosity of RHGOF as well as the higher accessible specific surface area.

At different current densities the charge-discharge profile of RHGOF/CNTs shown in Fig. 7b. It is found that all electrodes possess nearly a symmetric charge-discharge curve of triangular shapes, illustrating that the electrical double layer capacitance (EDLC) is contributed main part in the specific capacitance. The faint deviation from the rectangular shape of CV curves and the charge-discharge profiles of electrodes display a complex supercapacitance, called pseudo-capacitance and EDLC. The major cause of pseudo capacitance is due to the oxygenated groups that are presented on the surface of electrodes. It is considered that the oxidization or reduction of electrochemically active oxygenated groups is responsible to slightly distorted CV curves contain faint redox peaks (in XPS analysis) in the electrolyte ${ }^{41-43}$. At current density $0.5 \mathrm{~A} / \mathrm{g}$, the specific capacitance of the synthesized electrodes are 557, 329 and 208 F/g for RHGOF/CNTs, RHGOF and RGOF respectively are shown in Fig. 7a. 

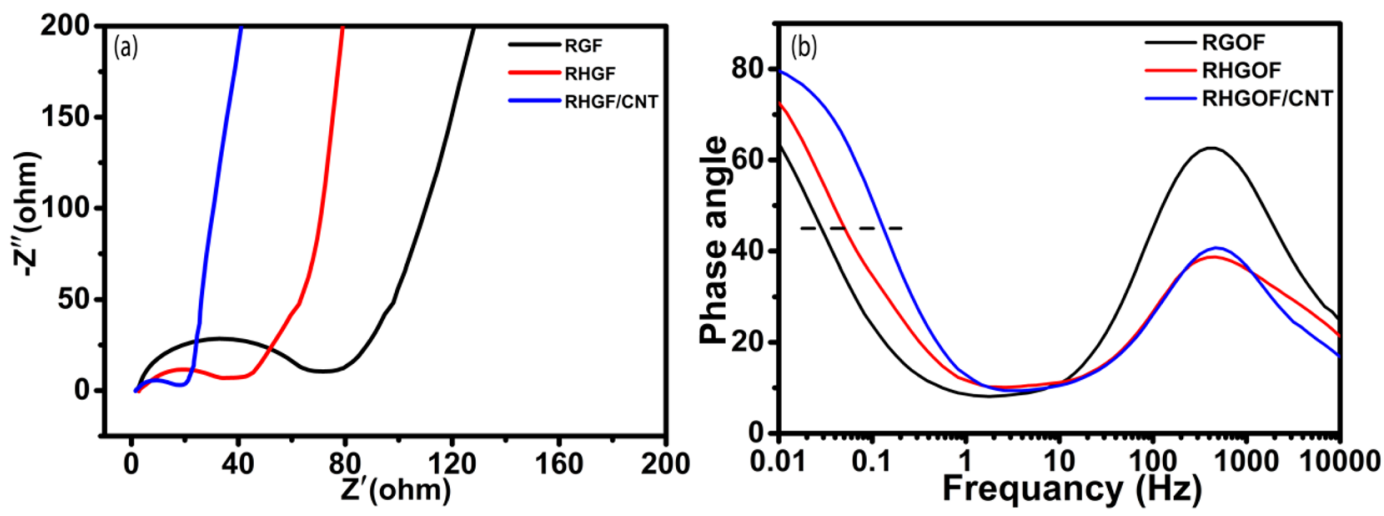

Figure 8. (a) EIS curves of RGOF, RHGOF and RHGOF/CNTs, (b) phase angle against frequency of RGOF, RHGOF and RHGOF/CNTscomposite.

The RHGOF/CNTs exhibits longest charge-discharge profile among all the electrodes is attributed to the highest capacitance, which shows similar pattern as in the CV curves. Furthermore, RHGOF/CNTs composite shows greater value than those of reported similar electrodes such as self-assembled reduced graphene oxide/carbon nanotubes thin film has specific capacitance $428 \mathrm{~F} / \mathrm{g}$ at $0.5 \mathrm{~A} / \mathrm{g}^{44}, \mathrm{GO} / \mathrm{CNT}$ sandwich paper has specific capacitance $151 \mathrm{~F} / \mathrm{g}$ at $0.5 \mathrm{~A} / \mathrm{g}^{45}$, holey GR paper (at $\left.1 \mathrm{~A} / \mathrm{g}, 201 \mathrm{~F} / \mathrm{g}\right)^{28}$, GR/mesoporous carbon nanosphere film electrode (at $0.2 \mathrm{~A} / \mathrm{g}, 211 \mathrm{~F} / \mathrm{g})^{46}$, multiwall carbon nanotubes/graphene film electrode (at $\left.0.1 \mathrm{~A} / \mathrm{g}, 265 \mathrm{~F} / \mathrm{g}\right)^{36}$ and CNT/holey graphene hybrid film (at $0.25 \mathrm{~A} / \mathrm{g}, 268 \mathrm{~F} / \mathrm{g})^{33}$, etc.

Figure 7 a shows the charge discharge profile of as prepared samples at $0.5 \mathrm{~A} / \mathrm{g}$ as the function of current density. The specific capacitance of RHGOF/CNTs is highest among all other electrodes. This highest capacitance can be attributed to the following considerations. At first, it is related to the porous network. A porous structure is supposed to produce pathways to electrolyte to penetrate deeply inside electrode and cut down the ion transport path. Hence, it significantly enhances the utilization of maximum area of RHGOF, as a result the conductive channel in the hybrid electrode is increased. Secondly, inside the RHGOF/CNT hybrid, CNTs acts as an electrically conductive connections, due to the diffusion of electrons and electrolyte ions inside the flexible electrode the average transport length of electrolyte ions is spectacularly shorten, hence charge-discharge process are facilitated during the reaction. Therefore, electrochemical as well as galvanostatic properties are enhanced. It is shown in Fig. 7c that the cyclic stability of RHGOF/CNTs is measured through the charge-discharge curve at $5 \mathrm{~A} / \mathrm{g}$. After 5000 consecutive charge discharge cycles, the capacitance retention RHGOF/CNTs is $90 \%$.

Figure 8a shows Nyquist plot of RGOF, RHGOF and RHGOF/CNTs electrode at $5 \mathrm{mV}$ signal voltage.

A close up view depicts that short $45^{\circ}$ Warburg region and semi circle for all three composites which are used as electrode. At high frequancy range equivalent series resistance (ESR) $R_{s}$ is evaluated by real part of resistance $\left(Z^{\prime}\right)$ with real axis provided by ionic resistance of the electrolyte, contact resistance with current collector and the intrinsic resistance of the active material. Charge transfer resistance $\left(\mathrm{R}_{\mathrm{ct}}\right)$ at the interface between electrolyte and electrode is shown by middle-high frequency range semi circle region. This is also known as Faraday's resistance. Figure 8a shows the semi circle of RGOF is largest than RHGOF and RHGOF/CNTs proving that RGOF has higher value of $R_{c t}$ than other two electrodes. The span of semi circle of RHGOF is reduced than RGOF as shown in the Fig. 8a. Similarly, span of semi circle of RHGOF/CNTs is reduced than RHGOF is attributed to increasing number of conductive sites to transport charges, which is increased by porous structure generation and CNTs growth. The plot of RHGOF/CNTs electrode shows fast ion diffusion and ideal capacitive behavior among the three electrode denoted by the sharpest slope during low frequency range. Fast ion diffusion took place in RHGOF/CNTs electrode due to the highly porous micro structure. Hence it is evidenced that the electrolyte is directly contact with active material electrode. As a result the distance of ionic diffusion is decreased.

From the Fig. $8 \mathrm{~b}$ it is shown that the dependence of impedance phase angle against the frequency for RGOF, RHGOF and RHGOF/CNT electrodes.All electrodes have discharge time with efficiency more than $50 \%$ is described as phase relaxation time constant $\left(\mathrm{t}_{\mathrm{o}}\right)$ of supercapacitor. We can find form the equation $\mathrm{t}_{\mathrm{o}}=1 / f_{\mathrm{o}}$ at phase angle of $-45^{\circ}$ with same capacitive and resistive impedance ${ }^{47}$. The characteristic frequencies are measured to be $0.046,0.082$ and $0.1778 \mathrm{~Hz}$ at phase angle of $45^{\circ}$ in $6 \mathrm{M} \mathrm{KOH}$ corresponding $\mathrm{t}_{\mathrm{o}}$ of $21.73,12.19$ and $5.62 \mathrm{sec}$ for RGOF, RHGOF and RHGOF/CNT, respectively. Smaller $t_{o}$ of RHGOF/CNTs shows that higher accessible surface area was available to electrolyte ions to improving electrical conductivity as compared to RGOF, and RHGOF. It was also shown that $t_{0}$ of RHGOF is smaller than RGOF, which evidences large accessible surface area that proved holes were present in the basal plane. In addition, due to very small relaxation time $t_{o}$ of RHGOF/CNTs this conductive 3D structure is responsible for dynamics of ion diffusion of electrolyte in the inner of electrode.

\section{Conclusion}

Sandwich RHGOF/CNTs hybrid material was successfully prepared by the combination of vacuum filtration and chemical vapor deposition. CNTs serve as conductive spacers in the RHGOF/CNTs structures, which prevent the restacking of RHGO layers. The 3D conductive interpenetrated architecture is perfect for fast ion penetration. The RHGOF/CNTs flexible composite is directly used as binder-free electrode. At $0.5 \mathrm{~A} / \mathrm{g}$, the current density of the RHGOF/CNTs hybrid reveals a high specific capacitance of $557 \mathrm{~F} / \mathrm{g}$. Moreover, the electrode displayed 
reasonable cycle stability and excellent rate capability.During the charge-discharge process this 3D structure is not only supportive to fast electron transfer but it is also beneficial in utilizing specific surface area to a greater extent. We thought that the flexible RHGOF/CNTs hybrid structure is a promising candidate for SECs electrodes.

\section{Experimental}

Synthesis of RHGOF, RGOF. Graphene oxide (GO) (XFNANO, Inc. Graphene oxide (GO) (XFNANO, Inc. Advance Material Supplier) was dispersed into ultra-pure water and treated under ultra-sonication for two hours ( $30 \mathrm{~min}$ sonication followed by $10 \mathrm{~min}$ stirring, consecutively) in water bath. Hence, a homogeneous suspension of GO $(1 \mathrm{mg} / \mathrm{ml})$ was obtained. Then, $0.4 \mathrm{ml}$ of $\mathrm{H}_{2} \mathrm{O}_{2}(30 \%)$ was mixed into GO suspension $(40 \mathrm{ml}, 1 \mathrm{mg} / \mathrm{mL})$ by continuous stirring for half an hour and we got a suspension. Afterwards $50 \mathrm{ml}$ Teflon lined autoclave was used for hydrothermal reaction. Finally autoclave was placed inside the oven for hydrothermal reaction at $100^{\circ} \mathrm{C}$ for 9 hours. Final product obtained was holey GO abbreviated HGO. To reduce the HGO, $0.67 \mathrm{ml}$ hydrazine solution (50\%) was poured into HGO suspension and after $5 \mathrm{~min}$ stirring as prepared HGO suspension is refluxed at $100^{\circ} \mathrm{C}$ for one and half an hour and cooled down naturally to the room temperature. As a result, after reduction HGO suspension changed into reduced holey graphene oxide solution and final solution was named as RHGO. Finally, reduced holey graphene oxide film (RHGOF) was prepared via vacuum filtration by using filter paper with pore size of $0.45 \mu \mathrm{m}$. As an experimental comparison, reduced graphene oxide film (RGOF) was synthesized by using the same conditions to prepare RHGOF, without adding $\mathrm{H}_{2} \mathrm{O}_{2}$.

Preparation of RHGOF/CNTs sandwich structure. CNTs were grown using the ferric salt $\left(\mathrm{FeCl}_{3}\right)$ as a precursor in formation of catalytic iron nano particles. $0.0135145 \mathrm{~g}$ of $\mathrm{FeCl}_{3}$ was dissolved in $50 \mathrm{ml}$ deionize water to prepared $0.001 \mathrm{M}$ catalytic solution. Afterwards, RHGOF was kept dipping in the prepared solution for 12 hours so that iron catalytic solution penetrates into the RHGOF. Than as prepared sample was dried at room temperature for 12 hours naturally. The CNTs were grown by using chemical vapor deposition (CVD) methods while $\mathrm{C}_{2} \mathrm{H}_{2}$ was used as carbon source. Catalyst carrying sample was placed into a quartz tube on a Si strip, and transferred into the center of CVD chamber. First the as prepared sample was heated at $710^{\circ} \mathrm{C}$ for $9 \mathrm{~min}$, while Ar $(470 \mathrm{sccm})$ atmosphere is provided before supplying carbon source. Afterwards a mixture of $\mathrm{C}_{2} \mathrm{H}_{2}(18 \mathrm{sccm})$ and $\operatorname{Ar}(457 \mathrm{sccm})$ was simultaneously introduced into the chamber for $5 \mathrm{~min}$ to grow CNTs. Finally, the sample was naturally cooled down to room temperature under the Ar atmosphere with a flow rate of $470 \mathrm{sccm}$.

Characterization of as prepared samples. The structural characterization of as prepared samples were characterized by a field emission scanning electron microscopy (QUANTA 450, 20 KV,FESEM), moreover morphology was also studied by using high-resolution transmission electron microscopy (JEOLJEM-200,HRTEM). The defects in the prepared samples were studied by using a Raman spectroscopy (Renishawin Via Plus, He- $\mathrm{Ne}$ laser, $532 \mathrm{~nm}, 50 \%$ laser power). The atomic composition and surface chemistry of as prepared sample was studied by an X-ray photo electron spectroscopy (Al-K X-ray source, XPS, ESCALAB 250). The pore size, accessible surface area and at relative pressure $\left(\mathrm{P}_{\mathrm{o}} / \mathrm{P}\right)$ adsorption data in the range of $0.05-0.20$ of the prepared sample was measured by using Brunauer-Emmett-Teller(BET) method. Mechanical strength was measured by using universal material testing machine (Yl-S370) with crosshead speed $1 \mathrm{~mm} / \mathrm{min}$.

Received: 27 June 2019; Accepted: 31 December 2019;

Published online: 11 February 2020

\section{References}

1. Simon, P. \& Gogotsi, Y. Materials for electrochemical capacitors. Nature Materials 7, 845 (2008).

2. Stoller, M. D. et al. Graphene-Based Ultracapacitors. Nano Letters 8(10), 3498-3502 (2008).

3. Lee, S. et al. LEGO-like assembly of peelable, deformable components for integrated devices. Npg Asia Materials 5, e66 (2013).

4. Miller, J. R. \& Simon, P. Electrochemical Capacitors for Energy Management. Science 321(5889), 651 (2008).

5. Burke, A. R\&D Considerations for the Performance and Application of Electrochemical Capacitors. Electrochimica Acta Vol. 53, 1083-1091 (2007).

6. Zhang, L. L. \& Zhao, X. S. Carbon-based materials as supercapacitor electrodes. Chemical Society Reviews 38(9), 2520-2531 (2009).

7. Wang, G., Zhang, L. \& Zhang, J. A review of electrode materials for electrochemical supercapacitors. Chemical Society Reviews 41(2), 797-828 (2012)

8. Geim, A. K. Graphene: Status and Prospects. Science 324(5934), 1530-1534 (2009).

9. Novoselov, K. S. et al. A roadmap for graphene. Nature 490, 192 (2012).

10. Geim, A. K. \& Novoselov, K. S. The rise of graphene. Nature Materials 6, 183 (2007).

11. Zhu, Y. W. et al. Graphene and Graphene Oxide: Synthesis, Properties, and Applications. Advanced Materials 22(35), 3906-3924 (2010).

12. Sun, Y., Wu, Q. \& Shi, G. Graphene based new energy materials. Energy \& Environmental Science 4(4), 1113-1132 (2011).

13. Zhang, H. et al. Self-generating graphene and porous nanocarbon composites for capacitive energy storage. Journal of Materials Chemistry A 3(21), 11277-11286 (2015).

14. Allen, M. J., Tung, V. C. \& Kaner, R. B. Honeycomb Carbon: A Review of Graphene. Chemical Reviews 110(1), 132-145 (2010).

15. Wu, D. et al. Nanocomposites and macroscopic materials: assembly of chemically modified graphene sheets. Chemical Society Reviews 41(18), 6160-6177 (2012).

16. Huang, X. et al. Graphene-based composites. Chemical Society Reviews 41(2), 666-686 (2012).

17. Luo, J., Kim, J. \& Huang, J. Material Processing of Chemically Modified Graphene: Some Challenges and Solutions. Accounts of Chemical Research 46(10), 2225-2234 (2013).

18. Chen, Q. et al. MnO2-modified hierarchical graphene fiber electrochemical supercapacitor. Journal of Power Sources 247, 32-39 (2014).

19. Zhou, X. et al. Bamboo-like Composites of V2O5/Polyindole and Activated Carbon Cloth as Electrodes for All-Solid-State Flexible Asymmetric Supercapacitors. ACS Applied Materials \& Interfaces 8(6), 3776-3783 (2016).

20. Linlin, L. et al. A Flexible Quasi-Solid-State Asymmetric Electrochemical Capacitor Based on Hierarchical Porous V2O5 Nanosheets on Carbon Nanofibers. Advanced Energy. Materials 5(17), 1500753 (2015). 
21. Gao, H. et al. Flexible All-Solid-State Asymmetric Supercapacitors Based on Free-Standing Carbon Nanotube/Graphene and Mn3O4 Nanoparticle/Graphene Paper Electrodes. ACS Applied Materials \& Interfaces 4(12), 7020-7026 (2012).

22. Xie, J. et al. Layer-by-layer $\beta-\mathrm{Ni}(\mathrm{OH}) 2 /$ graphene nanohybrids for ultraflexible all-solid-state thin-film supercapacitors with high electrochemical performance. Nano Energy 2(1), 65-74 (2013).

23. Cheng, H. et al. Textile electrodes woven by carbon nanotube-graphene hybrid fibers for flexible electrochemical capacitors. Nanoscale 5(8), 3428-3434 (2013).

24. Yan, Z. et al. Three-Dimensional Metal-Graphene-Nanotube Multifunctional Hybrid Materials. ACS Nano 7(1), 58-64 (2013).

25. Zhuangjun, F. et al. A Three-Dimensional Carbon Nanotube/Graphene Sandwich and Its Application as Electrode in Supercapacitors. Advanced Materials 22(33), 3723-3728 (2010).

26. Xiong, C. et al. Two - step approach of fabrication of three - dimensional reduced graphene oxide - carbon nanotubes - nickel foams hybrid as a binder - free supercapacitor electrode. Electrochimica Acta 217, 9-15 (2016).

27. Fan, W. et al. Flexible free-standing 3D porous N-doped graphene-carbon nanotube hybrid paper for high-performance supercapacitors. RSC Advances 5(12), 9228-9236 (2015).

28. Xu, Y. et al. Solution Processable Holey Graphene Oxide and Its Derived Macrostructures for High-Performance Supercapacitors. Nano Letters 15(7), 4605-4610 (2015).

29. Bai, Y. et al. Formation process of holey graphene and its assembled binder-free film electrode with high volumetric capacitance.Electrochimica Acta Vol. 187 (2015).

30. Zang, P. Y. et al. Green synthesis of holey graphene sheets and their assembly into aerogel with improved ion transport property. Electrochimica Acta 212, 171-178 (2016).

31. Shuangyan, G. et al. Polyaniline Nanorods Grown on Hollow Carbon Fibers as High-Performance Supercapacitor Electrodes. ChemElectroChem 3(7), 1142-1149 (2016).

32. Cheng, H. et al. Textile electrodes woven by carbon nanotube-graphene hybrid fibers for flexible electrochemical capacitors. Nanoscale 5(8), 3428-3434 (2013).

33. Deng, L. et al. Carbon nanotubes/holey graphene hybrid film as binder-free electrode for flexible supercapacitors. Journal of Colloid and Interface Science 494, 355-362 (2017).

34. Jiménez Riobóo, R. J. et al. Elastic constants of graphene oxide few-layer films: correlations with interlayer stacking and bonding. Journal of Materials Chemistry C 3(19), 4868-4875 (2015).

35. Byon, H. R. et al. Thin films of carbon nanotubes and chemically reduced graphenes for electrochemical micro-capacitors. Carbon 49(2), 457-467 (2011).

36. Lu, X. et al. A flexible graphene/multiwalled carbon nanotube film as a high performance electrode material for supercapacitors. Electrochimica Acta 56(14), 5115-5121 (2011).

37. Xiaowei, Y. et al. Bioinspired Effective Prevention of Restacking in Multilayered Graphene Films: Towards the Next Generation of High-Performance Supercapacitors. Advanced Materials 23(25), 2833-2838 (2011).

38. Chen, C.-M. et al. Hierarchically aminated graphene honeycombs for electrochemical capacitive energy storage. Journal of Materials Chemistry 22(28), 14076-14084 (2012).

39. Abdelkader, A. M. et al. Alkali Reduction of Graphene Oxide in Molten Halide Salts: Production of Corrugated Graphene Derivatives for High-Performance Supercapacitors. ACS Nano 8(11), 11225-11233 (2014).

40. Brun, N. et al. Design of Hierarchical Porous Carbonaceous Foams from a Dual-Template Approach and Their Use as Electrochemical Capacitor and Li Ion Battery Negative Electrodes. The Journal of Physical Chemistry C 116(1), 1408-1421 (2012).

41. Andreas, H. A. \& Conway, B. E. Examination of the double-layer capacitance of an high specific-area C-cloth electrode as titrated from acidic to alkaline pHs. Electrochimica Acta 51(28), 6510-6520 (2006).

42. Bichat, M.-P., Raymundo-Piñero, E. \& Beguin, F. High voltage supercapacitor built with seaweed carbons in neutral aqueous electrolyte. Carbon Vol. 48, 4351-4361 (2010).

43. Zhao, B. et al. Supercapacitor performances of thermally reduced graphene oxide. Journal of Power Sources 198, 423-427 (2012).

44. Huang, Z.-D. et al. Self-assembled reduced graphene oxide/carbon nanotube thin films as electrodes for supercapacitors. Journal of Materials Chemistry 22(8), 3591-3599 (2012).

45. Huang, Z.-D. et al. Effects of reduction process and carbon nanotube content on the supercapacitive performance of flexible graphene oxide papers. Carbon 50(11), 4239-4251 (2012).

46. Lu, X., Dou, H. \& Zhang, X. Mesoporous carbon nanospheres inserting into graphene sheets for flexible supercapacitor film electrode. Materials Letters Vol. 178 (2016).

47. Taberna, P. L., Simon, P. \& Fauvarque, J. . El Electrochemical Characteristics and Impedance Spectroscopy Studies of Carbon-Carbon Supercapacitors. Journal of The Electrochemical Society, 150(3), p. A292-A300 (2003).

\section{Acknowledgements}

This work was supported by the national science foundation of china. (NO. 51972039, 51661145025, 11274055) and LiaoNing revitalization talents program (NO. XLYC1902122).

\section{Author contributions}

Khan Abdul Sammed initiates the key idea and presented to Pan Lujun (PhD supervisor). Overall experiments and measurements were performed and designed by Khan Abdul Sammed. Muhammad asif guided regarding data acquisition and analysis. Muhammad Usman and Tianze Cong gave valuable contribution and shaping the manuscript with scientific technical discussion. Amjad Farid took part in sample preparations and critically revised the overall manuscript. Muhammad asif imran discussed the experimental arrangements and reviewed the manuscript. Pan Lujun was responsible for project planning and funding. Correspondence and requests for materials should be addressed to Pan Lujun. All authors discussed the results and implications and commented on the manuscript at all stages.

\section{Competing interests}

The authors declare no competing interests.

\section{Additional information}

Supplementary information is available for this paper at https://doi.org/10.1038/s41598-020-58162-9.

Correspondence and requests for materials should be addressed to L.P.

Reprints and permissions information is available at www.nature.com/reprints. 
Publisher's note Springer Nature remains neutral with regard to jurisdictional claims in published maps and institutional affiliations.

(c) (i) Open Access This article is licensed under a Creative Commons Attribution 4.0 International License, which permits use, sharing, adaptation, distribution and reproduction in any medium or format, as long as you give appropriate credit to the original author(s) and the source, provide a link to the Creative Commons license, and indicate if changes were made. The images or other third party material in this article are included in the article's Creative Commons license, unless indicated otherwise in a credit line to the material. If material is not included in the article's Creative Commons license and your intended use is not permitted by statutory regulation or exceeds the permitted use, you will need to obtain permission directly from the copyright holder. To view a copy of this license, visit http://creativecommons.org/licenses/by/4.0/.

(c) The Author(s) 2020 